\title{
Kirchen und Massenmedien nach den österreichischen Synoden
}

\author{
von Peter Pawlowsky
}

\section{Voraussetzungen und Geschichte}

"Trotz des Offentlichkeitscharakters der Kirche hat sie zuwenig geschulte Fachleute für die Massenmedien. Der personelle und finanzielle Einsatz, der für die Verkündigung auf den herkömmlichen Wegen ... aufgewendet wird, steht in einem krassen Mißverhältnis zum Aufwand für die mediale Verkündigung..." "Wer sich ... in den Massenmedien engagiert ... wird in einer Zeit knapper werdender Kräfte für die herkömmlichen offiziellen kirchlichen Aufgaben eher als Konkurrent empfunden. ${ }^{\text {"2 }}$ Solche Sätze aus den Texten des "Ósterreichischen Synodalen Vorgangs" (OSV = Osterreich-Synode) machen den Eindruck einer kritischen Situationsanalyse und zählen tatsächlich zu den schärfsten Formulierungen der österreichischen Synoden, soweit davon innerkirchliche Zustände betroffen sind. Dennoch: Vier Jahre, nachdem solche Sätze zu Papier gebracht, zwei Jahre nachdem sie mit bischöflicher Zustimmung veröffentlicht wurden, besitzen sie unverminderte Geltung. Der intensiven und jahrelangen Bemühung um eine Verbesserung des Verhältnisses zwischen Kirche und Massenkommunikation ist der entscheidende Durchbruch noch nicht gelungen. Es gibt Teilerfolge, es gibt ein besseres Klima. Das ist schon viel; aber ist es alles, was die Synoden zustandegebracht haben? Haben sie mehr erreicht, als eine Kodifizierung des bisher Erreichten? Sind sie nur Zusammenfassung oder auch Wende im Selbstverständnis der Kirche gegenüber den Massenmedien?

\subsection{Medienarbeit als Filmarbeit}

Die Überlegungen der Synoden haben nicht beim Nullpunkt angesetzt. Zwei Jahrzehnte kirchlicher Medienarbeit (wenn man nur die Zeit nach dem Krieg ins Auge $\mathrm{fa} \mathrm{Bt}$ ) liegen vor ihnen, ein weiteres Jahrzehnt ist seither in enger Verbindung und parallel zur synodalen Reflexion vorübergegangen. Nicht zufällig stand am Anfang die Filmarbeit. Nach einem Jahrhundert, in dem Medienarbeit für die Kirche gleich Pressearbeit war und diese im Sinn kirchlicher oder kirchennaher Presseproduktion verstanden wurde, ging man in der Beschäftigung mit dem Film erstmals davon aus, daß der Aufbau einer Gegen-Produktion nicht die einzige Form der christlichen Bewältigung des massenmedialen Angebots sein konnte. Die „Katholische Filmkommission für Osterreich" veröffentlicht seit ihrer Gründung (1947) Filmgutachten, die zuerst in der Nachrichtenagentur „Kathpress“, dann - seit 1951 - in einer eigenen Zeitschrift, der "Filmschau“" versuchte $\mathrm{zwar}$, die alten Intentionen des Presseapostolats auf das neue Medium anzuwenden, und sogar ein kleiner Ausflug in die Filmwirtschaft wurde riskiert (Stephanus-Verleih, Stephanus-Produktion). Zwischen 1949 und 1963 gelang in acht „Inter-

Dr. phil. Peter Pawlowsky ist Wiener Redakteur der katholischen Wochenzeitung "präsent" (Innsbruck) und als freier Mitarbeiter des ORF Redakteur und Präsentator zahlreicher religiöser TV-Sendungen. Er redigierte den Informationsdienst der Wiener Synode (19671971) und war bei der Wiener Synode wie beim Osterreichischen Synodalen Vorgang (OSV) Berichterstatter der Medienkommissionen vor den Synoden-Vollversammlungen. Seit 1974 ist er Vorsitzender des Zentrums für Massenkommunikation der Erzdiözese Wien. 
nationalen Festwochen des religiösen Films" noch die Sammlung und Präsentation des „guten " Films, aber von da an blieb es bei Filmkritik, "Katholischen Filmsonntagen" (1956 bis 1963) und filmerzieherischer Erwachsenenbildung. Noch 1972, zum 25jährigen Jubiläum der Filmkommission, überwog das Bedauern über diese Entwicklung: „Es gehört wohl zu den schmerzlichsten Erfahrungen und Erkenntnissen der Filmkommission“", konnte man damals lesen, "daß sie gegen das Uberhandnehmen übler und übelster Filme ... machtlos war ...; daß ihr also kein Einfluß auf die Produktion gelang, kein nennenswerter auf das Verleihwesen und sie sich letzten Endes mit Teilerfolgen auf dem Gebiet der Information von filminteressierten Menschen begnügen muß." 4

\subsection{Die neuen Medien}

Die kirchliche Beschäftigung mit Hörfunk und Fernsehen war nur insofern weniger produktionsorientiert, als in Osterreich nie ernsthaft die Errichtung einer eigenen katholischen Rundfunkanstalt erwogen wurde. Die Katholische Aktion gründete eine „Arbeitsgemeinschaft für Rundfunkfragen “ (1950), die zunächst neben der Filmkommission herlief und sich um die ersten Sendungen religiösen Inhalts kümmerte; Programmplanung und Sprecherauswahl für zugewiesene Sendezeiten geschahen im wesentlichen hier, und diese enge Zusammenarbeit mit dem Rundfunk ergab anders als es für die Filmkommission möglich war - einen starken Einfluß auf die Produktion. Mit der Arbeit von Filmkommission und Arbeitsgemeinschaft hatte die kirchliche Medienarbeit eine Stabilität gefunden, die bis in die Mitte der Sechzigerjahre reichte. Die Promulgation des Konzilsdekrets über die sozialen Kommunikationsmittel, „Inter mirifica“ (1963), worin nationale „Stellen für Presse, Film, Rundfunk und Fernsehen"s gefordert wurden, brachte erste Versuche einer organisatorischen Vereinheitlichung in Gang. $1965 \mathrm{kam}$ es zur Gründung des „Katholischen Zentrums für Film, Funk und Fernsehen", in dem je eine neue nach dem Muster der Filmkommission gegründete Hörfunk- und Fernsehkommission untereinander und mit der Filmkommission lose kooperierten. Das Konzil hatte das Medienwesen nicht dem Laienapostolat, sondern der direkten Leitung der Bischöfe zugewiesen; tatsächlich erfolgte die Neugründung gegen den Widerstand der Katholischen Aktion, die entgegen vorherigen Abmachungen - ihre „Arbeitsgemeinschaft für Rundfunk und Fernsehen" (diesen Namen hatte unterdessen die „Arbeitsgemeinschaft für Rundfunkfragen" angenommen) aufgeben mußte (1965/1966).

Für diese hatte sich schon seit 1961 durch die Gründung einer rundfunkinternen Hauptabteilung Kirchenfunk im Osterreichischen Rundfunk (ORF) die Situation verändert. Nun war die Katholische Aktion zugunsten einer noch enger als sie selbst an die Amtskirche gebundenen Institution aus der Medienarbeit ausgeschaltet. Weil umgekehrt hinter den drei Kommissionen des Zentrums keine Mitgliederbewegung stand, führten diese im großen und ganzen das Dasein statutarisch und finanziell abhängiger bischöflicher Fachgremien, denen aber die Tatsache der Kirchenunabhängigkeit der Medien einen gewissen Freiheitsraum sicherte.

\subsection{Beginn der synodalen Ära}

Damit sah sich die kirchliche Medienarbeit mit dem Beginn der österreichischen Synoden in eine institutionell ziemlich schwierige Position manövriert, die ihr auch in den synodalen Beratungen zu schaffen machte. Die Kommissionsmitglieder, eine kleine Gruppe von Journalisten, Rundfunkleuten und Priestern, war dem Mißtrauen der Laienorganisationen ebenso ausgesetzt wie der Skepsis der Hierarchie, 
suchte den Bedeutungsverlust der Filmarbeit zu verarbeiten und zugleich die Zusammenarbeit mit dem Rundfunk fortzuführen, ohne auf irgendeinen gesetzlichen Status hinweisen zu können - ganz abgesehen von den inneren Schwierigkeiten, auf die weiter unten noch einzugehen sein wird. In den knappen acht Jahren zwischen dem 15. September 1966 (Konstituierung der vorbereitenden Kommission „Kirche und soziale Kommunikation" der Wiener Diözesansynode) und dem 4. Mai 1974 (Verabschiedung des Medienpapiers der Osterreich-Synode) fand die kirchliche Medienarbeit ihre heutige Organisationsform, ohne die oben genannten Schwierigkeiten voll überwinden zu können. Der Fortgang der Dinge war von einer vielfachen Wechselwirkung von Impulsen des Zentrums an die Synoden und der Synoden an das Zentrum bestimmt. Die Wiener Synode hatte ihr erstes, vorläufiges Medienpapier schon 1967 zur Diskussion vorgelegt. Nach einem ersten Versuch von 1968 gelang 1969 eine Statutenreform des „Katholischen Zentrums für Film, Funk und Fernsehen“ mit der Tendenz zu besserer Zusammenarbeit und breiterer Fundierung. Im Februar 1970 wurde als letzte Medienkommission die „Katholische Pressekommission für Osterreich" gegründet. Ihr sollten die - im Vergleich zur Filmkommission - konträren Schwierigkeiten zum Verhängnis werden: Neben prominenten Journalisten gaben in der Pressekommission vor allem die katholischen Verleger und Publizisten, also die Produzenten, den Ton an; es stellte sich aber heraus, daß Einzelinteressen und Branchenkontakte zu stark waren, als daß die entscheidenden Absprachen in der Pressekommission der Bischöfe getroffen worden wären. Alles was in diesen Jahren im katholischen Pressewesen Osterreichs geschah oder nicht geschah - so die Verbesserung der Zusammenarbeit der Kirchenzeitungen oder der mißlungene Ausbau des Wochenblattes „Der Volksbote“ zu einer repräsentativen gesamtösterreichischen katholischen Wochenzeitung - fand außerhalb der Pressekommission statt. Ohne daß die KoInmission ihr Selbstverständnis gefunden hätte, legte ihr letzter Vorsitzender 1975 seine Funktion zurück.

\subsection{Das „Katholische Zentrum für Massenkommunikation"}

1970 allerdings erschien die Gründung der Pressekommission als Fortschritt und ermöglichte wenige Monate später den entscheidenden Schritt zur organisatorischen Zusammenfassung der kirchlichen Medienarbeit: Am 13. Juni 1970 fand die Gründungsversammlung des „Katholischen Zentrums für Massenkommunikation“ statt, in dem das bisherige „Zentrum für Film, Funk und Fernsehen“ aufging und das aus den vier Kommissionen für Film, Hörfunk, Fernsehen und Presse besteht. Neu war dabei neben der Hinzunahme der Presse vor allem die Schaffung eines arbeitsfähigen Vorstandes und Präsidiums bei immer noch erheblicher Selbständigkeit der Kommissionen und die Festlegung, daß analoge Zentren, ebenfalls bestehend aus je vier Medienkommissionen, in jeder Diözese zu errichten seien. Die laufenden Diözesansynoden wurden benutzt, um diese Forderung zu verankern. Das zeitliche Ineinandergreifen dieser Entwicklungen ist gewiß ein wichtiger Grund für die Behandlung der Medien in den österreichischen Synoden, zumal es sogar sozusagen personifiziert wirksam wurde: Anton Fellner, Generalsekretär der Wiener Diözesansynode, ein prominenter Journalist, bekleidete zugleich führende Funktionen im Zentrum, und Walter Schaffelhofer, der Sekretär der Osterreich-Synode, spielt eine wichtige Rolle in der österreichischen Medienpolitik ${ }^{8}$.

Schon 1968 stellte die erste österreichische Synode nach dem Konzil, die Salzburger Synode, einen Text zum Thema "Massenmedien" zur Diskussion, den die Synodalkommission „Verkündigung" erarbeitet hatte. 
Ein halbes Jahr vor dem Erscheinen der Pastoralinstruktion „Communio et progressio" (Mai 1971) verabschiedete im Oktober 1970 die Wiener Synode ihre Medienvorlage. Im März 1972 gab die Innsbrudker Diözesansynode pastorale Leitsätze zur Medienfrage heraus, im Mai 1972 wurde ein ausführlicher Text der Kärntner Synode zur „kirchlichen Kommunikation " promulgiert, im Herbst desselben Jahres folgte die St. Pöltner Synode mit ihrem Medienpapier. Damit hatten sich fünf der sieben nachkonziliaren österreichischen Diözesansynoden mit den Massenmedien befaßt. Die Synoden in Linz und Eisenstadt verzichteten darauf, und zwar schon im Hinblids auf den (gesamtösterreichischen) OSV. Die O'sterreich-Synode hatte ihre Kommission „Kirche und Massenmedien“ im April 1972 konstituiert und deren Texte im Oktober 1973 in erster, im Mai 1974 in zweiter Lesung angenommen. Darin wird das „Katholische Zentrum für Massenkommunikation" in seiner seit 1970 bestehenden Form schon vorausgesetzt. Dieses hatte unterdessen noch zwei wichtige Schritte tun können: Einmal gelang die Errichtung einiger, wenn auch nicht aller diözesanen Zentren für Massenkommunikation; zum anderen kam es mit Beginn 1973 zur Herausgabe einer neuen Zeitschrift des Zentrums unter dem Titel „multimedia - Zeitschrift für kritische Medienarbeit" "7. In dieses Blatt, das anfangs wöchentlich, heute vierzehntägig erscheint, gingen die hektographierten Informationsdienste der Hörfunk- und Fernsehkommission ebenso ein, wie - nach hinhaltendem Widerstand der Filmkommission - die traditionsreiche "Filmschau" in ihrem 23. Jahrgang.

\section{Probleme der Synodentexte}

Eine Analyse der synodalen Medientexte muß von dieser reichlich verworrenen innerkirchlichen Entwidklung ausgehen, um wenigstens einige ihrer Widersprïchlichkeiten verständlich zu machen. Zunächst nämlich erwiesen sich die von den Synoden in Gang gesetzten Beratungsprozesse als Gelegenheit zur Artikulation und Aufarbeitung von Spannungen, die innerhalb der kirchlichen Medienarbeit entstanden waren. Diese Spannungen sind in die Texte eingegangen und drücken sich auch nach vielen Überarbeitungen im Nebeneinander-Stehen unvermittelter Textstücke und - im Verlauf der Synodengeschichte - in extrem unterschiedlichen Abstimmungsergebnissen aus.

\subsection{Widersprüche aus der kirchlichen Medienarbeit}

Die Filmkommission hatte 1965, bei der Gründung des „Zentrums für Film, Funk und Fernsehen", unter Berufung auf "Inter mirifica “, aber auch durch längere Tradition und größere Nähe zur Amtskirche noch die Kraft gehabt, ihr Strukturmodell den beiden Kommissionen für die anderen Medien aufzuprägen. Als zwei Jahre später der erste Wiener Synodentext zu den Massenmedien herauskam ${ }^{8}$, wurde offenkundig, daß eine inhaltliche Integration der Arbeit in den verschiedenen Kommissionen des Zentrums nicht gelungen war. Noch deutlicher fiel die damals nicht einmal formal ins Zentrum eingebundene Presse aus dem Rahmen: Freiheit und Selbstverantwortung des katholischen Publizisten und Medienkonsumenten standen der Aufforderung gegenüber, die Gläubigen sollten ,sich an die kirchlichen Filmgutachten halten "9. Solche Widersprüche wurden zwar in späteren Fassungen der Vorlage abgeschliffen, verschwanden aber nicht völlig. Die Katholische Filmkommission war ja, wie oben dargestellt, im Laufe der Jahre immer stärker auf die Gutachtertätigkeit reduziert worden. Nun mußte sie gerade dort, wo sie selbst erfolglos geblieben war, nämlich bei der Einflußnahme auf die Produktion, mitansehen, wie durch die Hör- 
funk- und Fernsehkommission wenigstens im Bereich des Kirchenfunks neue und wirksame Formen der Produktionsmitgestaltung entwickelt wurden. Auch die früher vorwiegend moralischen - Maßstäbe der Beurteilung gerieten in Bewegung, und die Tatsache, daß für Hörfunk- und Fernsehsendungen fast nie genügend Informationen zur Bewertung im voraus zur Verfügung stehen, minderte das Gewicht kirchlicher Auswahlvorschläge überhaupt. Nimmt man hinzu, daß die elektronischen Medien in ihrer gesellschaftlichen Wirksamkeit den Film überflügelten, die Filmkommission aber durch Tradition, Budget und personelle Ausstattung immer noch den anderen Kommissionen überlegen war, so werden die zahlreichen Konflikte verständlich, die auf dem Weg zum gemeinsamen Zentrum für Massenkommunikation und daher auch in den synodalen Beratungen auszutragen waren.

\subsection{Meinungsschwankungen der Synoden}

In den Synoden fand die auf Bewahrung bedachte Haltung der Filmkommission im Bewußtsein vieler Synodalen ihre Entsprechung. Dem erwachenden Verständnis dafür, daß sich die Kirche „der Intensität der gesellschaftlichen Kommunikation nicht entziehen " kann" ${ }^{10}$, stand eine anhaltende Angstlichkeit vor der moralischen Schädlichkeit und den Manipulationsmöglichkeiten der Massenmedien gegenüber. Nur so konnte es etwa dazu kommen, daß im Oktober 1970 bei der zweiten Session der Wiener Synode ein Antrag auf völlige Zurückweisung der Medienvorlage gestellt wurde und nur mit knappen zwei Stimmen (131 zu 133) unterlag; dieselbe Vorlage aber, nachdem sie so knapp überlebt hatte, wurde noch am selben Tag mit geringfügigen Änderungen Absatz für Absatz mit Zwei-Drittel-Mehrheiten verabschiedet. Drei Jahre nachher, im Oktober 1973, lag der Osterreich-Synode die Vorlage „Kirche und Massenmedien" zur ersten Lesung vor. Bei der Abstimmung fand der Text so ungeteilte Zustimmung, daß der erste und einzige im strengen Sinn einstimmige Beschluß dieser Synode zustande kam: Es gab weder Nein-Stimmen noch Stimmenthaltungen.

Die Vorlagen unterscheiden sich inhaltlich zu wenig, um solche Differenzen in den Abstimmungsergebnissen zu erklären. Aber es hat sich immer wieder gezeigt, daß ein Synoden-Plenum dort, wo die Meinungsbildung nicht abgeschlossen ist oder tieferliegende Unsicherheiten weiterwirken, für aktuelle Stimmungsschwankungen besonders anfällig ist. "Gegner des vorgelegten Textes“, hieß es in einem Bericht über die Wiener Synodendebatte vom Oktober 1970, „kritisierten vor allem, daß zuviel von Freiheit die Rede sei und in der Vorlage auf die mit den Massenmedien verbundenen Gefahren zu wenig hingewiesen werde... Aktuelle Probleme wie die Bildung von Meinungsmonopolen, die Sexwelle in den Medien und die sich ausbreitende Pornographie lieferten Stoff für die Redner; der Vorlage wurde schließlich die ideologische Tendenz der Neuen Linken unterstellt ... "11

Liest man nach, welche Texte zu solcher Aufregung Anlaß gaben, so stößt man auf eine knappe, zum Teil lehrhafte Einführung in die Probleme der Massenkommunikation und auf Bekenntnisse zur Meinungsfreiheit, gegen Meinungsmonopole und Zensur. Die Kirche, lautet einer der deutlichsten Sätze, „sieht in der Kritik einen Dienst an der Gemeinschaft und folgert daraus auch für sich die Bereitschaft, der Kritik nicht feindselig, sondern mit der Einladung zum offenen Gespräch zu begegnen "12. Dementsprechend wird von einer Verpflichtung zur offenen Information gesprochen und vor den Gefahren einer Politik der Geheimhaltung gewarnt ${ }^{13}$, übrigens in ganz ähnlichen Worten, wie es einige Monate später "Communio et pro- 
gressio ${ }^{\alpha}$ tun sollte ${ }^{14}$. Die unzufriedenen Gegner der Wiener Medienvorlage wurden im Oktober 1970 durch die Einfügung eines neuen Leitsatzes beruhigt, der „den wirksamsten Schutz vor dem Mißbrauch der Freiheit" von Meinungsäußerung und Kritik ,in der persönlichen Verantwortung jedes einzelnen Mitarbeiters der Massenmedien " sieht ${ }^{15}$. Diesen Leitsatz hatte es schon in einem der vorbereitenden Papiere der Wiener Synode gegeben, doch war er von den Journalisten der Kommission als zwar richtig, aber unnütz empfunden worden. Als die Osterreich-Synode dreieinhalb Jahre später, im Mai 1974, die zweite Lesung über ihr Medienpapier hielt, entstand in der Versammlung wieder Unsicherheit über allzu deutliche Aussagen gegen kirchliche Zensur und für das Recht der freien Meinungsäußerung. Der Kommissionsvorsitzende erinnerte sich des oben zitierten Leitsatzes der Wiener Synode und schlug denselben Text zur Aufnahme in das Papier der Osterreich-Synode vor; dabei erwies diese Formel abermals ihre beruhigende Wirkung ${ }^{16}$.

\subsection{Pragmatik gegen Isolation}

Versucht man, dieses Durcheinander verschiedener Tendenzen und daraus entstandener Kompromisse und Ungereimtheiten zu ordnen, so zeichnet sich das Bild folgender Grundschwierigkeit ab: Einerseits ist die Forderung nach Medienfreiheit im außerkirchlichen Bereich zu stark, um ignoriert $\mathrm{zu}$ werden; anderseits sehen viele Christen den moralischen Niedergang des Films und der Illustrierten gerade als Ergebnis solcher Medienfreiheit an. Einerseits werden die Christen seit Jahren von ihren Hirten dazu angehalten, Medienfreiheit für die Kirche zu fordern und den nichtkirchlichen Medienerzeugnissen mit zunehmender Kritikfähigkeit zu begegnen; anderseits scheinen Lehramt und Tradition einer Medienfreiheit und Toleranz für Kritik innerhalb der Kirche enge Grenzen zu setzen. Diese Gegensätze sind durch das Konzil erst manifest geworden, und den Synoden ist es nicht gelungen, sie aufzulösen. So blieben nebeneinander und unverbunden stehen: überaus mutige Aussagen über die Freiheit der Medienschaffenden und der Medienkonsumenten und Appelle zur Verantwortung, die unkonkret sind und daher wirkungslos bleiben müssen. Solchen Appellen kann zwar nicht widersprochen werden, aber sie entlarven eine immer noch vorhandene Hilflosigkeit der Kirche im Umgang mit den Massenmedien und der Offentlichkeit überhaupt.

Vor diesem Hintergrund stellt sich die innerkirchliche Isolation, mit der die Mitarbeiter des Zentrums zum Teil noch heute zu kämpfen haben, als mehr dar denn ein vermeidbares Ergebnis des Konflikts mit der Katholischen Aktion und des nur langsam wachsenden Verständnisses von seiten der Hierarchie. Es hat vielmehr den Anschein, als würde von denen, die sich berufsmäßig in den Umgang mit den Massenmedien eingelassen haben, dort, wo sie für die Kirche tätig werden, ein Kirchenverständnis vorausgesetzt, das sich weder mit dem der Laienorganisationen noch mit dem der Hierarchie deckt. Hatte die Filmarbeit ihr Massenmedium noch als klares Gegenüber ansehen und beurteilen können, so erforderten die elektronischen Medien ein entschiedenes Hineingehen in die Strukturen des Rundfunks, also Einwirken als Mitwirken. Dieser Sachverhalt hat theologische Implikationen, für die es gewiß auch anderswo Ansätze gibt; aber da Medienleute meist keine Theologen sind und daher ihre eigenen theologischen Voraussetzungen nicht reflektieren, überwinden sie ihre Isolierung gewöhnlich nicht, sondern überspringen sie pragmatisch, weil die Arbeit in den Medien ihren permanenten Einsatz erfordert und sie anderseits von der Kirche gebraucht werden. Dem Beobachter bleibt aber nicht verborgen, daß sich hinter 
Gebrauchtwerden und Gewährlassen auch nach den Synoden häufig gegenseitige Verständnislosigkeit verbirgt. Auch die Beschlußfassung der Synoden über die Medienpapiere war allem Anschein nach das Ergebnis spontaner Koalitionen, in denen sich Verfechter dieses neuen, wie wir es unten nennen werden, kommunikativen Kirchenverständnisses mit anderen zusammenfanden, die lediglich von der instrumentalen Nützlichkeit der Massenmedien für die Kirche beeindruckt waren.

\section{Die Frage nach dem theologischen Ort}

Pragmatik ist die Stärke der Texte. Eine Fülle konkreter Anregungen, die in den nächsten Jahren verwirklicht werden sollen, sind von den Medienfachleuten zusammengetragen und von den Synodalen beschlossen worden (vgl. dazu die Zusammenstellung im Anhang). Die Widersprüche im Grundsätzlichen hinderten die Synoden nicht an der Beschlußfassung über praktische Regelungen, im Gegenteil: Pragmatik wurde bisweilen zur Flucht aus dem Dilemma. Erst im Abstand wird deutlich, daß die Ungelöstheit der Grundfragen weiterwirkt und nun ihrerseits die Verwirklichung der Synodenbeschlüsse, und zwar der ganz praktischen, behindert. Grundsätzliche und theologische Überlegungen haben auch in anderen Zusammenhängen immer wieder die Langeweile und den Mißmut der Synodalen geweckt; im Falle des Verhältnisses von Kirche und Massenmedien sind sie heute notwendiger denn je, weil die zeitgeschichtlichen Verwicklungen der kirchlichen Medienarbeit, wie sie oben in (1.) und (2.1.) skizziert wurden, die Schwierigkeit dieses Verhältnisses nicht ausreichend erklären. Vielmehr bedarf die Geschichte an dieser Stelle selbst einer Interpretation durch die Erhebung des theologischen Bewußtseins, das sich in ihr manifestiert hat.

Ansatzpunkte für theologische Fragestellungen sind in den Vorlagen der Wiener Synode und - sehr viel ausführlicher - der Osterreich-Synode (OSV) zu finden. Innsbrudk beschränkt sich auf eine grundsätzliche Bejahung der Massenmedien, verpflichtet die Amtsträger, die Gläubigen „zum rechten Gebrauch“ der Medien zu erziehen, und die Gläubigen, von diesen „wählend und unterscheidend Gebrauch zu machen ${ }^{\alpha 17}$. Die St. Pöltner Synode holt weiter aus und bedient sich vieler Formulierungen von "Communio et progressio"; gegenüber der Auseinandersetzung um Freiheit oder Bevormundung, die die Einleitungspassagen der Wiener Texte prägt, tritt die unersetzliche Bedeutung der Massenmedien für die Verkündigung in den Vordergrund $^{18}$, eine Tendenz, die sich im spätesten Papier, jenem der Osterreich-Synode, noch stärker durchsetzte. Die Salzburger und die Kärntner Synode stellen ihre Medienvorlage überhaupt in das Kapitel "Verkündigung" und verzichten - abgesehen von einigen Zitaten aus Konzilstexten und aus "Communio et progressio" (letzteres nur in Kärnten, da Salzburg schon 1968 abschloß) - so gut wie vollständig auf jede theologische Motivation der kirchlichen Beschäftigung mit den Medien.

\subsection{Einheit als Kommunikation}

Dort, wo nach dem theologischen Ort der Massenkommunikation gefragt wird, schiebt sich vor die eigentliche Auseinandersetzung mit dieser Frage die allgemeine nach dem theologischen Stellenwert von Kommunikation überhaupt. "Weil Gott sich selbst den Menschen mitgeteilt und sie dadurch in Gemeinschaft mit ihm gebracht hat, fühlt sich die Kirche allem verbunden, was Kommunikation schaff ${ }^{\boldsymbol{\alpha} 19}$, so beginnt das Mediendokument der Wiener Synode. Offenbarung, als gemeinschafts- 
stiftendes Ereignis der Kommunikation verstanden, wird im Text der OsterreidSynode unter den Stichworten "Kommunion statt Kommunikation?" noch deutlicher entfaltet: „Die Verbindung der Kirche mit ihrem Herrn ist der Grund ihres Einheit. Kommunion und Kommunikation können daher in der Kirche nicht gut voneinander getrennt werden. Der Hinweis, daß in Christus alle Glieder und Gemeinden der Kirche am tiefsten verbunden seien, bleibt eine Phrase, wenn er nidht als Anspruch aufgefaßt wird, diese Verbindung greifbar zu realisieren: In der vollen, uneingeschränkten, über alle Spannungen hinweg aufrechterhaltenen Kommunikation innerhalb der Kirche und mit der Welt kann die Kirche ihre Kommunion mit dem Herrn am besten glaubhaft machen. ${ }^{\text {"20 }}$

Den Begriff Kommunikation hatte zuvor schon "Communio et progressio" in das Bedenken der Selbstmitteilung Gottes in Jesus und der Einheit der Kirche in der Eucharistie eingeführt ${ }^{21}$. Daß dabei Einheit nur im Prozeß der Kommunikation gegeben verstanden werden kann, geht noch klarer aus jenen Formulierungen hervor, die die Uberschrift „Kommunikation oder Ex-Kommunikation“ tragen: „Das öffentliche Interesse und die Tatsache, daß sich über die Amtsträger hinaus immer mehr Christen am Leben und an der Meinungsbildung der Kirche beteiligen, machen die Ubberschaubarkeit und Leitung der Kirche immer schwieriger. Das Unvermögen, eine Ordnung von hoher Komplexität aufrechtzuerhalten, weil in ihr der Platz des einzelnen ungewiß geworden ist, verleitet nicht nur die Amtsträger, sondern gleichermaßen den einzelnen Christen und verschiedenen Gruppen in der Kirche immer wieder zum Versuch, die allseitige Kommunikation soweit abzubrechen, daß die je eigenen Meinungen und Ansprüche auf markierten Wegen durchgesetzt werden können. Störende Einflüsse werden ex-kommuniziert. Die Folge davon ist aber, daß an den Abbruchstellen Stauungen entstehen: Während sich die Kirche auf diesem Weg vorläufige Ruhe sichert, weil sie sich der permanenten Reform entzieht, wachsen solche Stauungen zu einer weit größeren Gefahr für die Ordnung des kirchlichen Lebensvollzugs an. “"22 Noch vor dem instrumentalen Verständnis von Massenkommunikation als Vehikel christlicher Verkündigung ist also, nach diesen Texten, Kommunikation ein Konstitutivum kirchlichen Lebensvollzugs. Von hier aus würde sich auch ein theologischer Zugang zum Verständnis der Massenkommunikation eröffnen und - nun nicht mehr einfach instrumental - der Verkündigung durch diese.

Tatsächlich entwirft die Osterreich-Synode noch ein kommunikatives Konzept kirchlicher Lebensvorgänge in den drei Schritten: Rezeption - Meinungsbildung Reform. "Laufende Veränderungen der Situation erfordern immer neue Entscheidungen; diese sind jeweils Entscheidungen zur Reform oder zum Aufschub der Reform. Soll eine Reform Aussicht auf Erfolg haben, so muß die Entscheidung zu ihr bereits von einer möglichst umfassenden Meinungsbildung getragen werden... Mit der Durchführung beginnt neuerlich ein Prozeß der Kommunikation, in dem die Praxis einer laufenden Korrektur unterzogen wird, weil Reaktionen und Erfahrungen aller Betroffenen bereits Elemente einer neuen zur Reform führenden Meinungsbildung sind. "23 Einheit der Kirche in diesem Sinn als Kommunikationsprozeß verstanden, eröffnet die Möglichkeit, Freiheit der Meinungsäußerung, Kritik, selbstverantwortete Entscheidung und Abschaffung jeder Form von Zensur als innerste Notwendigkeiten kirchlichen Lebens zu begreifen. Denn „Meinungsmonopole bestimmter kirchlicher Gruppen, seien es auch die Amtsträger, und die Verhinderung der Kritik bedeuten Kommunikationsabbruch und führen zu einseitigen, für das Ganze der Kirche schädlichen Entscheidungen. ${ }^{24}$ Auch eine solche Aussage ist nur vor dem Hintergrund 
eines Kirchenverständnisses möglich, in dem Einheit nicht statisch, sondern im dynamischen Vorgang des Austausches auf allen Ebenen menschlicher Existenz erscheint, leibhaft wird, und das heißt sich in greifbaren, darum auch angreifbaren Strukturen organisiert. Das soll im folgenden verkürzt ein kommunikatives Kirchenverständnis genannt werden; es ist als Bedingung einer sachgerechten Beschäftigung der Kirche mit den Massenmedien anzusehen, wobei sachgerecht zugleich mediengerecht und theologisch verantwortet meint.

\subsection{Ansehen und Absicherung}

\subsubsection{Das Argument „Rückwirkung“}

Eine gründliche theologische Aufarbeitung müßte hier erst beginnen: Unter dem Gesichtspunkt der Kommunikation wären der Verkündigungsauftrag jedes Getauften und Gefirmten ${ }^{25}$, wären die Aufgaben des Lehramts und der Kirchenleitung neu zu formulieren. Aber die Synoden haben diesen Anlauf in ihren eigenen Texten nicht durchgehalten, sondern immer wieder versucht, die Problematik der Massenkommunikation mit vordergründigen Argumenten einerseits, mit Absicherungbewegungen anderseits zu bewältigen. So gibt es einen ganzen Block von Aussagen, die unter dem Stichwort „Rückwirkung “ zusammengefaßt werden können. Nachdem etwa Kritik als „Dienst an der Gemeinschaft ${ }^{\text {“ }}$ gewürdigt wurde, folgt der Satz: „Dies gilt um so mehr für kritische Äußerungen aus den eigenen Reihen, soll die Haltung der Kirche nicht unglaubwürdig werden.“" ${ }^{28}$ Oder: „... wirksame und möglichst störungsfreie innerkirchliche Kommunikation [ist] Voraussetzung für die Glaubwürdigkeit der Kirche nach außen. ${ }^{427}$

Kommunikatives Verhalten in der Kirche und die Schaffung kommunikativer Strukturen der innerkirchlichen Meinungsbildung erscheinen nach solchen Aussagen als Rüdkwirkung säkularer Entwicklungen, denen sich die Kirche notgedrungen anpaßt, um nicht in den Ruf der Rückständigkeit zu geraten. Entgegen dem einleitenden Entwurf ist hier die Perspektive eines kommunikativen Kirchenverständnisses wieder aus den Augen verloren. An ihre Stelle tritt der Versuch, kommunikative Verhaltensweisen zur Konsolidierung der eigenen Glaubwürdigkeit aus der Gesellschaft zu übernehmen, also mit Anpassung Ansehen zu erwerben und sich auf diesem Weg Wirkungsmöglichkeiten zu sichern. Das erklärt, daß Massenkommunikation weiterhin als Fremdeinfluß empfunden wird. Es ist in der Folge nur konsequent, wenn man dagegen wieder Barrieren aufrichtet. So formulierte etwa die Osterreich-Synode: „Bewußtes Totschweigen, Zensur und Redeverbot sind ungeeignete Methoden zur Herstellung gemeinsamer Uberzeugungen "; aber die Ơsterreichische Bischofskonferenz fügte, ehe sie den Text promulgierte, hinzu, „daß dem innerkirchlichen Dialog dort Grenzen gesetzt sind, wo Glaube und Sitte bewußt gefährdet werden "28. Die Bewegung im Gegensatz von Anpassung und Abgrenzung führt nicht weiter. Hier gilt nicht mehr, daß das, was in der Kommunikation - auch über die Massenmedien vermittelt wird, zur Auseinandersetzung herausfordert und „in einem dynamisch fortschreitenden Kommunikationsprozeß selbst wieder Gegenstand der Information und Kritik" werden muß ${ }^{29}$; hier gilt auch nicht, daß die Kirche "in einem Klima des geistigen Pluralismus... - im Vertrauen auf die Überzeugungskraft der Botschaft Jesu - den Widerstreit der Meinungen und die offene Information weder in der Offentlichkeit noch im eigenen Wirkungskreis zu scheuen ${ }^{\text {" }}$ braucht $^{30}$; vielmehr ist der dialektische Ablauf des Kommunikationsprozesses in ein stïckweises Hintereinander von zugelassenem Dialog und vorgeschriebener Grenzziehung zerlegt, gesteuert von 
einer kirchlichen Autorität, die sich ihrer eigenen Einbindung in den Kommunika, tionsprozeß nicht bewußt ist.

\subsubsection{Mandat für Verkündiger?}

Dem gewählten Beispiel könnten viele zur Seite gestellt werden; es soll nicht dea Eindruck erwecken, als hätte dieses Problem seinen Ort allein in Differenzen zwischen den Synoden und der Hierarchie. Das Bedürfnis nach Absicherungen, seien sie. auch inhaltsleer, gegen die Gefahr, in den Strudel der Kommunikation hineingezogen zu werden, war durchaus in den Synoden selbst lebendig. Manche der Appelle zur "persönlichen Verantwortung jedes einzelnen “s1 gehören hierher. Dort, wo es im engeren Sinn um Verkündigung in den Massenmedien geht, wird die Angst sogar ausgesprochen: Es fehle „häufig an der mediengerechten Gestaltung, weil befürchtet wird, der Inhalt der Botschaft könnte verändert werden, wenn man versucht, sie auf neue Weise zur Sprache zu bringen "32. Es waren solche Befürchtungen, gegen die ein Leitsatz des gesamtösterreichischen Medienpapiers formuliert wurde, der den Satz enthält: „Bei der ausdrücklichen Verkündigung in den Massenmedien kommt dem zentralen Inhalt der Botschaft ein klarer Vorrang zu. "${ }^{\text {"33 }}$ Solche Ratschläge aus dem Plenum der Synode helfen im Grunde nicht, aktivieren aber verständliche Bedürfnisse nach Absicherung bei denen, die Verkündigung in den Massenmedien versuchen und deren Mühe nicht nur oft unbedankt bleibt, sondern mit Anfeindungen aus der Kirche selbst bedacht wird. "Während allgemein in der Verkündigung an Ausbildung und Autorisierung ihrer Träger notwendigerweise strenge Maßstäbe angelegt werden, steht der in den Medien Verkündende oft in einem schweren persönlichen Dilemma: Im Falle von Meinungsverschiedenheiten wird seine grundsätzliche Verkündigungsberechtigung als Getaufter und Gefirmter im Hinblick auf die Wirksamkeit der Medien als unzureichend erklärt, auch wenn die nötige Ausbildung gegeben ist, so daß den Trägern der Verkündigung in den Massenmedien mangels eines festumschriebenen Mandats oft der notwendige Rückhalt fehlt. "

Die schmerzlichen Erfahrungen, die hinter diesem Satz stehen, führten zum Versuch einer theologischen Differenzierung des Verkündigungsgeschehens. Zunächst wird festgehalten, daß Informationen aus dem Leben der Kirche und Verkündigung klar getrennt werden müssen, daß also die Verkündigungsabsicht ein Schönfärben von Informationen keineswegs rechtfertigt. „Die Informationsarbeit in der Kirche und für die Kirche steht gerade dann im Dienste der Verkündigung, wenn sie sich nicht als Verkündigung mißversteht. ${ }^{\text {"35 }}$ Sodann erscheint nach dem Medientext der Osterreich-Synode Verkündigung in zweifacher Hinsicht als „abgestuftes Geschehen“: Einmal was die Ausdrücklichkeit betrifft, die „von der direkten Bezeugung bis zur Enthaltenheit in allen Vorgängen der zwischenmenschlichen Kommunikation, der Wissensvermittlung, des wissenschaftlichen Erkennens und künstlerischen Schaffens reicht ${ }^{{ }^{38}}$; zum anderen hinsichtlich des Inhalts, dessen Relevanz je nach der Nähe zur Person und dem Werk Jesu „von absoluter Verbindlichkeit bis zum Angebot von Rat und Beispiel reicht“s7. Daraus wird gefolgert: „Je ausdrücklicher ... Verkündigung ist, je relevanter ihre Inhalte sind und je mehr Offentlichkeit ihr zukommt, umso qualifizierter müssen Ausbildung und Autorisierung des Verkündenden für seine Aufgabe sein. ${ }^{\text {"s8 }}$

\subsection{Eine Verkündigungsdidaktik fehlt}

Diese Unterscheidungen sind so interessant wie abstrakt. Sie führen zu keinen praktikablen Regeln etwa für den Einsatz von Sprechern in kirchlichen Sendungen oder für 
Konflikte mit dem Lehramt. Um so mehr kann das Bedürfnis, sie im Synodentext zu verankern, als Zeichen für die Spannungen angesehen werden, die auch heute noch im Bereich der medialen Verkündigung $\mathrm{zu}$ bewältigen sind. Dazu kommt die ungeklärte Frage, welches Ziel solche Verkündigung eigentlich habe, da sie doch nicht unmittelbar gemeindebildend sein kann. Die Österreich-Synode spricht in diesem Zusammenhang von der "Bereitung eines geistigen Vorfeldes" ${ }^{\text {"39, }}$, sowie davon, ,die Botschaft Jesu zu einem Bestandteil des durch die Medien vermittelten ,Weltbildes" [zu] machen und damit Glauben [zu] ermöglichen und [zu] fördern ${ }^{\alpha 40}$. Wie soll das geschehen? Die Wiener Synode entwickelte schon einige Jahre vorher Gesichtspunkte für mediengerechte Verkündigung; denn „im Hinblick auf die Massenmedien ist die Heilsbotschaft neu zu durchdenken und so zu präsentieren, daß sie sich in die Eigenart des jeweiligen Mediums einfügt ${ }^{\alpha 41}$. Dabei wird zweierlei hervorgehoben: Einmal „soll die Botschaft spontan aufgegriffen und weitergetragen werden $^{\alpha}$, worunter die Wahl eines aktuellen Ansatzpunktes und die Forderung verstanden werden, daß Sprache und Argumentation weder kompliziert noch autoritär sein dürfen. Zum anderen wird davon gesprochen, daß „die Fülle des Anzubietenden in viele kleine geistige Einheiten $\mathrm{zu}$ teilen" seit2 $^{42}$ und somit nur "scheinbar systemlos" ist; eine „kluge Lenkung [möge] dafür sorgen, daß - im ganzen und auf Zeit gesehen - die gesamte Heilsbotschaft vermittelt wird ${ }^{443}$. Solche Hinweise können für die mediale Verkündigung in ihrer ausdrücklichsten Form, also etwa für die eigentlich religiösen Sendungen gelten, und auch hier nur, soweit es um die Methode der Darbietung geht. Inhaltliche Uberlegungen, schon gar für das oben zitierte „Vorfeld“, fehlen fast ganz.

Wenn man von dem absichernden Hinweis auf den Vorrang des zentralen Inhalts der Botschaft ${ }^{44}$ absieht, nimmt nur ein einziger kurzer Text - ein Leitsatz der Osterreich-Synode - auf die Themenwahl Bezug und spricht damit das didaktische Problem an: "Die Gestalter kirchlicher Medienarbeit müssen das Evangelium auch dort als befreiende Botschaft glaubhaft machen, wo ein konsequentes christliches Leben im Gegensatz zu eingeschliffenen Zuständen in der Gesellschaft steht. Solche Gegensätze auszusprechen, ist notwendig und bieter Ansatzpunkte für die Themenwahl in der Verkündigung. "45 Damit mag ein Hinweis auf die gesellschaftskritische, vielleicht auch prophetische Funktion einer Verkündigung in den Massenmedien gegeben sein; aber dieser Leitsatz bleibt ein erratischer Block und findet nirgends im Text Vorbereitung oder Fortsetzung. Das bedeutet, daß es den Synoden nicht gelungen ist, den theologischen Ansatz eines kommunikativen Kirchenverständnisses zu einer Didaktik medialer Verkündigung weiterzuentwidkeln, d. h. sich mit der Frage nach der Relevanz der Verkündigungsinhalte auseinanderzusetzen.

Die Verpflichtung, sich der Massenmedien, da es sie nun einmal gibt, für die Verkündigung zu bedienen, konnte die Angst vor einer Veränderung der Botschaft durch das Medium nicht ausräumen, weil beide - Angst und Verpflichtungsgefühl (wie oben: Abgrenzung und Anpassung) - den inneren Zusammenhang von Kirche und Kommunikation, somit auch Massenkommunikation, nicht im Auge haben. Eine Verkündigungsdidaktik dürfte heute zu den dringendsten Aufgaben theologischer Beschäftigung mit den Massenmedien gehören; sie müßte die Verkündigungsinhalte im Hinblick auf Medien, Situation und Zielgruppe bedenken und damit das bloß instrumentale Verständnis der Medien von innen her überwinden. Die besondere Schwierigkeit liegt darin, daß diese Aufgabe nicht nur für die Verkündiger, sondern auch für die Masse der Hörer, Seher und Leser, besonders für die „schon Bekehrten“, 
zu leisten wäre; denn nur eine einer solchen medialen Verkündigungsdidaktik entsprechende "theologische Medienerziehung" könnte verhindern, daß die Mißverständnisse auf seiten jener erhalten bleiben, die die Botschaft in ihrer medialen Auswahl und Darbietung sozusagen nicht entschlüsseln können und daher nicht wiedererkennen.

\subsection{Innere Hindernisse}

Dieses Problem entsteht ja vor allem für jene, die auch ohne Medien von der kirchlichen Verkündigung erreicht werden. Mehrfach betonen die Synoden aber die große Reichweite der Verkündigung durch die Massenmedien; „für unzählige Menschen sind die Massenmedien der einzige Weg, auf dem ihnen die Heilsbotschaft nahegebracht werden kann. ${ }^{\text {} 48}$ Wen redet die Kirche also über die Medien an? Und wen erreicht sie? Hier liegen Unklarheiten, gibt es Hindernisse, und es ist evident, daß nicht alles zum Besten steht. Die Ursachen dafür sind oben aufgezählt worden: Vordergründig waren Spannungen in der kirchlichen Medienarbeit (2.2.) und eine gewisse kirchenpolitische Isolation derselben (1.2.; 1.3.) festzustellen, beides von einem Vierteljahrhundert Geschichte zugleich geschaffen und zum Ausdruck gebracht (1.). Das dahinterstehende Bewußtsein beruhte zwar stückweise auf einem kommunikativen Kirchenverständnis (3.1.), ließ sich aber immer wieder von der Nötigung zur Praxis (2.3.) bestimmen oder blieb auf halbem Weg im Gegensatz zwischen Anpassung und Abgrenzung (3.2.) stecken. Schließlich stellte sich heraus, daß die Neufassung der Inhalte einer medialen Verkündigung erst noch eine bevorstehende Aufgabe ist (3.3.). In den Synodentexten kommen alle diese Motive zu Wort, durchkreuzen und überlagern einander. Insofern bieten sie ein getreues Abbild des herrschenden Bewußtseins, das zugleich Möglichkeit und Hindernis kirchlichen Umgangs mit den Massenmedien ist. Das hat charakteristische Konsequenzen.

\subsubsection{Selbstbehinderung}

Die oben angedeutete Unklarheit, wer nun eigentlich über die Medien angeredet wird, ist eine solche Konsequenz. Adressaten der kirchlichen Verkündigung über die Medien sind zwar der Intention nach die der Kirche Fernstehenden, in der Praxis aber, wenn man Diktion und Themenwahl prüft, häufig doch die Nahestehenden. Denn die Gestalter medialer Verkündigung sind nicht nur selbst meist kirchliche Insider, sondern sie müssen sich - das ist viel entscheidender - auch vor jenem Teil der Hörer-, Seher- und Leserschaft verantworten, der selbst dem inneren Kreis der Praktizierenden angehört. Diese Rezipienten kennen nämlich die Verkündigungsinhalte aus Katechese und Liturgie, also aus ganz anderen Vermittlungsvorgängen, und protestieren, wenn das Bekannte für sie in der medialen Verkündigung nicht zu erkennen ist. Damit behindern häufig gerade die Engagierten selbst jenen missionarischen Elan der Verkündigung, den sie von der Kirche zu Recht fordern.

Unter den Gegenständen, mit denen sich die österreichischen Synoden beschäftigt haben, gibt es noch einige andere Beispiele für diese Schwierigkeit: die Pastoral an Fernstehenden, Arbeitnehmern und Jugendlichen sowie die Caritas-Arbeit. Daß den Synoden in den erstgenannten Problemkreisen keine wirklich weiterführenden Aussagen gelungen sind, ist ebenfalls das Ergebnis einer Selbstbehinderung: Es ist nämlich gar nicht so schwierig festzustellen, innerhalb welcher Lebensformen fernstehende Gruppen angetroffen und daher (wenigstens zunächst) akzeptiert werden müssen, und wie sie daher anzusprechen sind; weitaus schwieriger ist es, denen, die enger an 
die Kirche gebunden sind, zu vermitteln, daß auch diese Weise des Ansprechens noch die unverfälschte Heilbotschaft enthalten kann.

\subsubsection{Grenzüberschreitung}

Daraus ergibt sich: Kirchliche Medienarbeit ist dort blockiert, wo auch die Pastoral an den Fernstehenden immer wieder stecken bleibt. Mehr noch: Eine engagierte kirchliche Medienarbeit, die bewußt die Bekehrung der schon Bekehrten hinter sich lassen will, deckt die wunden Punkte der traditionellen Pastoral auf; wo es der Verkündigung über die Medien wirklich gelingt, Fernstehende anzusprechen, fühlen sich daher die Nahestehenden allzu oft verunsichert und zu Abwehrreaktionen provoziert. In diesem größeren Zusammenhang gesehen sind die Medientexte der österreichischen Synoden verschieden weit, zum Teil aber bis an die Grenzen des herrschenden kirchlichen Bewußtseins vorgestoßen und spiegeln dessen tieferliegende ungelöste Probleme. Umgekehrt haben Journalisten und Medienfachleute in den Synoden nicht nur ihrer Sache auf die Beine geholfen, sondern einen wichtigen Beitrag zur Veränderung des Bewußtseins in Richtung auf ein kommunikatives Kirchenverständnis geleistet - mit allem, was das für Verkündigung und Pastoral bedeutet. Trotz vieler Widerstände ist die Beschäftigung mit Kommunikation in den Synoden schon deswegen nicht ganz folgenlos geblieben, weil der Beratungsvorgang der Synoden selbst Grundzüge eines kommunikativen Kirchenverständnisses impliziert. Wenn dennoch von einem Durchbruch noch nicht gesprochen werden kann, so ist auch das Teil eines größeren Problems, das nicht allein im Spannungsfeld Kirche - Massenmedien bewältigt werden kann. Es handelt sich um das Problem von innen und außen, von Ghetto und Vorfeld, von Abgrenzung und Grenzüberschreitung, von Kirche und Welt und damit um das Grundproblem des Konzils und der nachkonziliaren Reformen. Die Massenmedien sind eine Stelle des Grenzübertritts, und im Zusammenhang mit ihnen haben die österreichischen Synoden auch die Aufgabe der Kirche reflektiert: Wenn für die Kirche das Kommunizieren der Botschaft Jesu Vorrang vor den Ansprüchen der Selbsterhaltung hat, dann auch Kommunikation vor Kommunikationsabbruch, Information vor Geheimhaltung, Meinungsfreiheit vor Zensur und vor allem: die Grenzüberschreitung zu den Fernstehenden vor den Bedürnissen der schon Bekehrten. Anders verfehlt die Kirche ihre Aufgabe, und was es heißt, sich in den gesellschaftlichen Kommunikationsprozeß nicht oder nur zögernd und mit Sicherung des Rückzugs einzulassen, zeigt sich an Folgen in allen Bereichen des kirchlichen Lebens.

\section{Medienpolitische Perspektiven}

Die eingangs gestellte Frage, ob die Synoden nur Zusammenfassung oder auch Wende im Selbstverständnis der Kirche gegenüber den Massenmedien gebracht haben, kann aus dem Material der Synoden nicht eindeutig beantwortet werden. Die Situation ist vielschichtig und fließend und hat sich auch seit den Synoden verändert. Wohin die Entwicklung aus dieser Phase des Übergangs läuft, wird man daran ablesen können, wie die anstehenden Probleme in den nächsten Jahren gelöst werden oder liegen bleiben. Daß Bewußtseinsveränderungen in der Kirche nötig sind, konnte oben ausführlich dargestellt werden; wie sie sich medienpolitisch auswirken könnten, soll im folgenden abschließend skizziert werden. 


\subsection{Die Stellung des „Zentrums“}

Durch die Synoden ist es gelungen, die Existenz des "Katholischen Zentrums für Massenkommunikation “ bzw. der diözesanen Zentren innerkirchlich bekannter zu machen, zumal dem Zentrum ausdrücklich eine Fülle von Aufgaben zugewiesen wurde. Schon in den Synodenpapieren kann man lesen, daß für die Erfüllung dieser Aufgaben (vgl. dazu die Zusammenstellung im Anhang) „ein entsprechender Ausbau des Zentrums unbedingte Voraussetzung " ist ${ }^{47}$. Dennoch ist es, wenn man die Geschichte kennt, kein Zufall, daß das größte kirchliche Projekt, das in den letzten Jahren außerhalb der Kirchenfunkabteilungen im ORF verwirklicht wurde, nicht der Initiative des "Zentrums“ entsprang. Das ORF-Studioprogramm "Wozu glauben?" (Januar bis März 1974), mit dem erstmals religiöse Bildungsinhalte in die Serie der ORF-Studienprogramme Eingang fanden, ist das Ergebnis von Bemühungen der Katholischen Aktion und ihres direkten Kontakts mit dem Rundfunk; in Vorbereitung und Durchführung war zwar die Bundesarbeitsgemeinschaft für katholische Erwachsenenbildung (BAKEB) eingeschaltet, nicht aber das „Zentrum“. Das Studienprogramm war ein beachtlicher Erfolg, weil es mit Hilfe der Gliederungen der Katholischen Aktion gelungen war, die größte Teilnehmerzahl aller bisherigen ORFStudienprogramme auf die Beine zu bringen und mit dazugehörigem Buch, Gesprächskreisen und Gruppentagen erstmals in der katholischen Erwachsenenbildung Medienverbund im großen Stil zu praktizieren. Innerkirchlich hat die Katholische Aktion damit nach fast einem Jahrzehnt ihren Anspruch geltend gemacht, kirchliche Medienarbeit und Medienpolitik mitzubestimmen. Damit muß in Zukunft gerechnet werden, d.h. das "Zentrum" kann kein Monopol in Sachen Medienarbeit für sich postulieren. Zugleich könnte die neue Zusammenarbeit dem "Zentrum" endgültig aus seiner innerkirchlichen Isolation heraushelfen. Denn auch ein umgekehrter Monopolanspruch der Katholischen Aktion ist längst nicht mehr möglich; das "Zentrum konnte seine Stellung nicht nur durch die Synoden festigen, sondern - insbesondere seit der Rundfunkreform von 1974 - auch medienpolitisch wirksam verankern: Der derzeitige Präsident des „Katholischen Zentrums für Massenkommunikation“, Franz Stauber, ist zugleich Vertreter der Kirchen im Kuratorium des ORF ${ }^{48}$ und bekleidet damit eine entscheidende Position am Schnittpunkt kirchlicher Interessen und öffentlicher Medienpolitik. Ein Zeichen für die Aufwertung des „Zentrums" und für künftige Chancen der Zusammenarbeit ist denn auch die Planung des zweiten religiösen ORF-Studienprogramms, das unter dem Titel "Wem glauben?" Anfang 1977 im Hörfunk laufen wird. Auch dafür stammte die Initiative von der

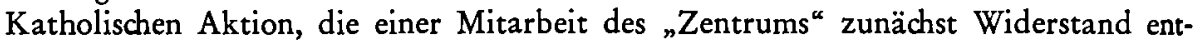
gegensetzte, schließlich aber einer gleichberechtigten Position zustimmte; heute ist das "Zentrum" neben der BAKEB die dritte Trägerorganisation in der Planung des ORF-Studienprogramms.

Die veränderte Situation, die sich an diesem Beispiel aufzeigen läßt, wird für die Zukunft von ausschlaggebender Bedeutung sein. Denn nur in einem Teamwork der Organisationen wird auch das „Zentrum“ seine Aufgaben erfüllen können. Es findet sich nach den Synoden in einer neuen Landschaft kirchlicher Organisationsstrukturen vor. Die Errichtung der pastoralen Räte von der Pfarre bis zur Diözese hat die seit 30 Jahren gültige Vorrangstellung der Katholischen Aktion im kirchlichen Engagement der Laien relativiert. Andererseits ist es nicht die Aufgabe einer an einem Sachbereich orientierten Institution, eine laienapostolische Bewegung zu organisieren. Schon jetzt zeigt sich, daß in Pfarrgemeinderäten, Vikariats- und Diözesanräten 
eine sachliche Zusammenarbeit zwischen Vertretern der Katholischen Aktion, der anderen katholischen Gruppen und Verbände und der Fachinstitutionen, deren eine das "Zentrum“ ist, die früheren Spannungen ablöst. So bietet sich für das „Zentrum “ erstmals die Chance, die für seine Medienarbeit notwendige Bewußtseinsbildung in größerem Rahmen voranzutreiben. Das bedeutet auf gesamtösterreichischer und diözesaner Ebene, daß die Bereiche Ausbildung, Forschung und Planung (vgl. dazu den Anhang) mit größerer Aussicht auf Erfolg bearbeitet werden können. Was von den vielen Vorschlägen der Synoden durchsetzbar und finanzierbar sein wird, nicht zuletzt daran wird man ablesen können, wie weit einem kommunikativen Kirchenverständnis der Durchbruch gelingt. Auf der Ebene von Pfarre und Dekanat wird dafür der „Medienreferent" unerläßlich sein, der nach der Vorstellung des „Zentrums" und der Synoden der kleinste lokale Stützpunkt der kirchlichen Medienarbeit sein soll. Unter den Beschlüssen der Osterreich-Synode ist diesbezüglich zu lesen: „Die Offentlichkeitsarbeit der Kirche - speziell im Dienste wechselseitiger Information - ist in geeigneten Stufen bis hinunter zur Pfarrbasis zu organisieren. $\mathrm{Zu}$ diesem $\mathrm{Z}_{\text {weck }}$ sind Medienreferenten in Vikariats-, Dekanats- und Pfarrgemeinderäten zu bestellen, die zur Erfüllung ihrer Aufgaben von den diözesanen Zentren und Informationsstellen laufend geschult und betreut werden. ${ }^{{ }^{49} 9}$ Bis heute gibt es solche Medienreferenten erst sporadisch. Sie werden für die oben beschriebene Bewußtseinsbildung eine wichtige Funktion haben; aber schon ihre zu erhoffende Existenz ist zunächst eine Frage des innerkirchlichen Bewußtseinsstandes in Sachen Kommunikation und Massenmedien und erst sekundär ein organisatorisches und finanzielles Problem.

\subsection{Bevorstehende Aufgaben in den Medien}

Die österreichische Medienlandschaft darzustellen und die nachsynodalen Aufgaben der Kirche daran zu formulieren, wäre ein eigenes und umfangreiches Vorhaben; an dieser Stelle müssen daher thesenartige Andeutungen genügen. Sie sollen folgenden vier Problemkreisen gelten: 1. die Situation Kirche - Presse als Ergebnis vieler Versäumnisse, 2. das ambivalente Verhältnis Kirche - ORF, 3. Vorbereitung auf die neuen Bild-Ton-Medien, und 4. die offene Frage nach den gültigen Kriterien in Medienkritik und Medienerziehung. In diese Richtungen werden in den nächsten Jahren Antworten gefordert sein.

Der katbolischen Presse ist hierzulande die Mentalität des Presseapostolats zum Schicksal geworden. Seit Jahrzehnten wird übersehen, daß der Rückgang der Meinungspresse auch deren Verbreitungsmodus obsolet macht: Gewissensnötigung zur Unterstützung „der guten Sache ${ }^{\star}$ kann in einer Zeit äußerlicher Unangefochtenheit der Kirche die mangelnde Qualität von Aufmachung und Inhalt nicht wettmachen. Es gibt heute eine wichtige Tageszeitung aus einem katholischen Preßverein ("Kleine Zeitung“, Graz und Klagenfurt), keine repräsentative gesamtösterreichische katholische Wochenzeitung ("präsent", der frühere Innsbrucker "Volksbote“, hat den Sprung in die große Offfentlichkeit nicht geschafft, die Wiener „Furche“ kränkelt), 11 Kirchenzeitungen mit relativ hohen, aber sinkenden Auflagen und eine wuchernde Wildnis kleiner und kleinster katholischer Vereins- und Ordensblätter, alles in allem ca. 440 Publikationen, dazu noch zwischen 1100 und 1600 Pfarrblätter $^{50}$. Die Zerstörung dieser publizistischen Infrastruktur zugunsten eines Konstrukts auf oberer Ebene wäre nicht nur unmöglich, sondern auch verfehlt. Denn die Situation der katholischen Presse in Osterreich zeigt, daß kommunikatives Bewußtsein, das 
zur Außerung drängt, in überschaubaren Strukturen da ist, im großen Rahmen aber fehlt. Das läßt sich aber nur durch einen langfristigen und über den Bereich der Presse hinausreichenden Prozeß des Umdenkens verändern.

Die Zusammenarbeit zwischen Kirche und ORF funktioniert wie vieles in Osterreich ohne gesetzliche Festlegung nach stiller Obereinkunft. Das Gesetz schreibt nur eine angemessene Berücksichtigung der gesetzlich anerkannten Kirchen und Religionsgemeinschaften im Programm vor. Aber in der Erstellung auch des Kirchenfunkprogramms ist der ORF rechtlich autonom. Die Planungsarbeit der Katholischen Hörfunk- und der Katholischen Fernsehkommission für das Programm ist auf unveränderte politische und personelle Verhältnisse angewiesen und entwickelt daher selbst die einem solchen System immanenten Tendenzen zur Stabilität mit geringer Neigung zu weiterführenden Experimenten. Da das System dennoch nicht garantiert ist, müßte es benützt werden, um so viel Raum für schöpferische Kräfte von seiten der Kirche frei zu machen, daß auch Experimente gewagt und Veränderungen ausgehalten werden können.

Die Vorbereitung auf die neuen Bild-Ton-Träger (Kassette und Bildplatte) schienen 1974 zu Ende der Osterreich-Synode aktueller als heute. Dennoch sollte das damals ausgearbeitete Konzept ${ }^{51}$ nicht in der Schublade bleiben. Die Entwicklung geht so schnell weiter - das zeigt etwa die gegenwärtige Diskussion über das Kabelfernsehen - daß Überraschungseffekte die kirchlichen Bemühungen um Mitgestaltung vereiteln können. Angesichts des personellen und finanziellen Aufwands, den eine Produktionsbeteiligung erfordert und der für viel bescheidenere Vorhaben kirchlicher Medienarbeit fehlt, können sich solche Pläne leicht als Illusion erweisen. Die nächsten Jahre werden zeigen, ob die kirchliche Medienarbeit auf diesem Gebiet das Schicksal der Filmarbeit wird teilen müssen.

Medienerziebung und Medienkritik werden eine zentrale Aufgabe bleiben und müssen noch ausgebaut werden. „Die Bemühung um die Fähigkeit zur umfassenden Kritik und Bewertung der Qualität des Angebots der Massenmedien ist ständige Aufgabe jedes Katholiken, die durch die kirchliche Medienarbeit gefördert und unterstützt werden muß." Bei kritischen Stellungnahmen nist vom Ganzen jenes Welt- und Menschenbildes auszugehen, das sich an der christlichen Botschaft orientiert, und eine Konfrontation mit allen Aspekten des Angebots zu versuchen, um einseitige Bewertungen zu vermeiden " ${ }^{52}$. Solche Sätze richten sich gegen eine moralische Verengung des Urteils, bieten aber darüber hinaus kaum einen Ansatz zur Entwicklung von heute tragfähigen Kriterien kirchlicher Medienkritik und Medienerziehung. Auch sonst bleiben die Synoden zu dieser Frage stumm; sie ist eine der schwierigsten in den Wandlungen der kirchlichen Medienarbeit der letzten Jahrzehnte und wird es allem Anschein nach bleiben. Eine Auseinandersetzung mit ihr wäre ein gesondert zu behandelndes Thema. Hier soll nur auf eine Gefahr hingewiesen werden: Wie das Fehlen einer Verkündigungsdidaktik den Ausweg aus dem Gegensatz von Anpassung und Abgrenzung versperrt (vgl. oben 3.3.), so auch der Mangel von Kriterien den Ausweg aus dem Gegensatz zwischen aufgedrungener Toleranz und dem Festhalten an unveräußerlichen Prinzipien. Durch die verschärft einsetzende Debatte um die Pornographie verhärten sich solche Gegensätze; es könnte sein, daß in den nächsten Jahren das Einnehmen von Abwehrpositionen in der Kirche wieder verstärkt zum guten Ton gerechnet wird. Sollte das der Fall sein, so könnte darunter die weitere Entfaltung eines kommunikativen Kirchen- 
verständnisses leiden und die Wirkung der Synoden in Frage gestellt werden. Noch ist die Entwicklung offen, noch steht der Durchbruch aus.

\section{Anhang: Ubersicht über die konkreten Vorschläge der Synoden}

Die folgende Zusammenstellung will keine vollständige Wiedergabe der Synodenbeschlüsse sein, sondern einen Überblick über die wichtigsten, oft gleichlautenden konkreten Vorschläge vermitteln, die in fünf Diözesansynoden und in der Österreich-Synode auf dem Gebiet der Massenmedien gemacht wurden. Das Material läßt sich nach folgenden sechs Gesichtspunkten einigermaßen gliedern:

\subsection{Finanzielle Mittel $^{1}$}

Fast durchwegs wird die Forderung nach besserer Dotierung der kirchlichen Medienarbeit erhoben. Die diözesanen und das gesamtösterreichische Zentrum sollen nideell, personell und materiell ausreichend " unterstützt werden, ebenso die diözesanen Informationsstellen und die „Kathpreß“. Mehr Geld wird insbesondere für den Nachwuchs (Stipendien) und für die Durchführung von Forschungsvorhaben auf dem Gebiet des Verhältnisses von Kirche und Massenmedien verlangt. Wichtig scheint die Forderung, Kirchenzeitungen auszubauen, statt von ihren Erträgnissen abzuschöpfen, oder wenigstens solche Erträgnisse wieder der Medienarbeit zuzuführen. Besonders hohe Beträge würde eine künftige Einschaltung der Kirche in das Kassetten- bzw. Bildplatten-Geschäft erfordern. Aber noch ohne überhaupt so weit zu denken, hat die OOsterreichische Bischofskonferenz, beeindruckt von der Vielzahl finanzieller und institutioneller Forderungen der Synoden, eine Fülle von Beschlüssen der Osterreich-Synode durch den Hinweis relativiert, daß diese Forderungen "von den einzelnen Diözesen und von der Bischofskonferenz nur im vorgegebenen Budgetrahmen erfüllt werden" können. Davon sind aus der Medienvorlage zwölf Beschlüsse betroffen.

\subsection{Stellen und Personen ${ }^{2}$}

Alle Synoden weisen die wichtigsten Aufgaben im Bereich der Medienarbeit den Zentren für Massenkommunikation zu und fordern dementsprechend deren Ausbau, ebenso die Errichtung diözesaner Presse- und Informationsstellen; diese Forderung ist übrigens schon fast überall erfüllt, allerdings haben die Pressereferenten in den verschiedenen Diözesen sehr unterschiedlichen Status. Ihnen werden auch Aufgaben der innerkirchlichen Kommunikation zugewiesen, ihre Zulassung zu Sitzungen der diözesanen Gremien und ihre offene Information durch die Amtskirche verlangt. Auf nationaler Ebene schlug die Osterreich-Synode eine zwischen Bischofskonferenz und „Kathpreß“ situierte Informationsstelle vor, die - gemäß „Communio et progressio" 175 - mehr als die Aufgaben einer Agentur wahrzunehmen hätte. Darüber hinaus soll sich ein eigener gesamtösterreichischer "Ausschuß für 'Offentlichkeitsarbeit" , der inzwischen errichtet, aber noch nicht mit dem verlangten Budget ausgestattet wurde, um Werbung und Offentlichkeitsarbeit für die Kirche kümmern und diese auf die pastorale Planung abstimmen.

Die wichtigste organisatorische und personelle Erfindung der Synoden unterhalb der diözesanen Ebene ist der Medienreferent. Er soll, wenigstens im Rahmen des Vikariats und der Dekanate, hauptberuflich angestellt werden, auf Pfarrebene jedenfalls Mitglied des Pfarrgemeinderats sein. Da in ihm alle Aufgaben der Medienarbeit 
zusammenlaufen, wird immer wieder auf die notwendige Ausbildung für ihn verwiesen. Unterschiede fallen hier im Text der Innsbrucker Synode auf, die anstelle von Medienreferenten mit offenbar eingeschränkteren Aufgaben Informationsverantwortliche in den Pfarrgemeinderäten und - auf der nächsten Ebene - vom Dekan (Dechanten) ernannte Berichterstatter vorsieht.

Schließlich bleiben in diesem Zusammenhang einige ganz konkrete Vorschläge der Synoden zu erwähnen: Es ist von einem zentralen diözesanen Postfach, sozusagen als Briefkasten für Anregungen und Beschwerden, die Rede; mehrere jährliche Pressekonferenzen des Bischofs zu feststehenden Terminen sollen die kontinuierliche Information der Offentlichkeit durch die Medien fördern; einmal jährlich soll eine Sonderausgabe der Kirchenzeitung oder eine Postwurfsendung allen Kirchenbeitragszahlern kostenlose Information ins Haus liefern.

\subsection{Material und Technik ${ }^{3}$}

Auf dem Gebiet der audio-visuellen Medien gibt es für die Kirche einen großen Aufholbedarf. Die Katholische Filmkommission soll die Einsatzmöglichkeiten von Kurzfilmen in der Verkündigung prïfen, wodurch sich ihr ein ganz neues Aufgabengebiet eröffnet. Zukunftsorientiert ist die Forderung nach einem eigenen Filmbzw. TV-Studio zur Úbungsproduktion. Die diözesanen Bildstellen, die meistenteils in der Entwicklung bei Diapositiv und Tonbildreihe stehen geblieben sind, sollen in die "Zentren für Massenkommunikation" eingegliedert werden und dort erweiterte Aufgaben bekommen. Insbesondere ist an die Aufnahme und Uberspielung von ORF-Sendungen gedacht, die in Katechese und Erwachsenenbildung eingesetzt werden können. Ein zentraler kirchlicher Schmalfilmverleih, der bisher an mangelndem finanziellen Einsatz gescheitert ist, soll das Angebot ergänzen. Um es auch nützen zu können, müssen möglichst viele Pfarren mit Wiedergabegeräten ausgestattet sein. Salzburg fordert einen Tonbildprojektor für jede Pfarre, was der Realität weir vorauseilt; der Verwirklichung noch ferner ist die bei den späteren Synoden verlangte Ausstattung mit Videorekordern. Im Zusammenhang mit dem Kassettenfernsehen entwirft die Osterreich-Synode die Vorstellung eines Abonnement-Ringes zur Anschaffung von Videorekordern und zum Vertrieb der Kassetten; ein eigenes Studio in kirchlichem Besitz zur Produktion solcher Kassetten wird in Erwägung gezogen. Von hier aus könnten auch dem ORF Angebote gemacht werden.

\subsection{Wissenschaft und Forschung ${ }^{4}$}

Den Einsatz wissenschaftlicher Methoden und Erkenntnisse verlangen die Synoden hinsichtlich der Medienarbeit in dreifacher Richtung: Einmal geht es einfach um die Berücksichtigung der Ergebnisse von Psychologie und Soziologie in der Verkündigung und um die Verwendung von Erhebungen und Umfragen bei der Beantwortung der Frage, wie mediale Verkündigung überhaupt ankommt. Solche Methoden werden zweitens für die Durchleuchtung der innerkirchlichen Kommunikation verlangt und sollen Informationsfluß und Stellen von Informationsstockungen sichtbar machen sowie Einstellung und Struktur der Zielgruppen bis auf die Ebene der Pfarre hinunter erheben. Schließlich sollen Kommunikationswissenschaft und $\mathrm{Me}$ dienkunde auf den theologischen Lehranstalten und Fakultäten eine Heimstätte bekommen (vgl. dazu auch 5.6.); das „Zentrum" soll einen Forschungsplan vor- 
egen, in dem diese Vorhaben koordiniert werden und der ihnen eine Finanzierung Jurch alle österreichischen Diözesen sichern soll.

\subsection{Planung und Zusammenarbeit ${ }^{5}$}

Der Ruf nach Planung ist eine der Antworten auf die divergierenden Tendenzen in Jer kirchlichen Medienarbeit. Geplant soll einerseits werden, was in den Medien über sinen längeren Zeitraum hinweg an Verkündigungsinhalten angeboten wird; anderseits sollen die Medien selbst - soweit sie kirchlichem Einfluß unterliegen - einer Planung und Durchforstung zugänglich gemacht werden. Wien entwarf ein „publizistisches Konzept", für ganz Osterreich soll die - zur Zeit (März 1976) inexistente - Katholische Pressekommission ein Pressekonzept erstellen. Schließlich wird das "Zentrum" mit der Erstellung eines langfristigen medialen Arbeitsplanes beauf tragt, der auf einen ebensolchen Pastoralplan des Osterreichischen Pastoralinstituts abgestimmt werden soll.

So weit gesteckte Ziele setzen aber zunächst einfachere Formen der Zusammenarbeit voraus, an denen es durchaus bis heute mangelt. Zusammenarbeit wird - dem eigenen Arbeitsprinzip der Synoden entsprechend - in vielen Zusammenhängen gefordert; sie soll, was die Medienarbeit betrifft, für eine bessere Koordinierung mit der Seelsorge die Voraussetzungen liefern und wird immer wieder als besondere Form der innerkirchlichen Kommunikation dargestellt. Ausdrücklich wird die $\mathrm{Zu}-$ sammenarbeit der Kirchenzeitungen über die Diözesangrenzen hinweg befürwortet - wozu auch schon einiges geschehen ist. Anders sieht es im innerbetrieblichen Raum aus: Der Wunsch, kirchliche Betriebe überhaupt zu Modellbetrieben zu entwickeln, in denen Formen von Mitbestimmung experimentiert werden, konkretisiert sich im Zusammenhang mit den Massenmedien in der Forderung nach Redaktionsstatuten für Kirchenzeitungen. Hier dürften noch große Hindernisse zu überwinden sein, so daß auf größere Kooperationsvorhaben wie den oben genannten Presseund medialen Arbeitsplan nicht so bald zu hoffen ist.

Allerdings bleibt an der Basis noch eine Menge zu tun. Die Synoden fordern die Kommunikation der Pfarren untereinander, nicht nur über die nächsthöhere Ebene. Dementsprechend ist von gemeinsamer Offentlichkeitsarbeit mehrerer Pfarren die Rede und werden gemeinsame Pfarrblätter ganzer Regionen dringend empfohlen. Überdies wird eine Konzentration katholischer Vereins- und Lokalblätter gefordert, was allerdings an den oben angedeuteten Schwierigkeiten, Pressepläne zu entwerfen, bislang gescheitert ist.

\subsection{Aus- und Weiterbildung ${ }^{6}$}

In der Sorge um Nachwuchs und Weiterbildung drückt sich die Einsicht in die minimalen personellen Reserven der kirchlichen Medienarbeit aus. Medienerziehung wird für die Schulen verlangt, soll in Knabenseminaren und Priesterseminaren zur normalen Ausbildung gehören und in den Studienplan aller Theologiestudenten, insbesondere der Religionslehrer eingebaut werden. Auch in der außerschulischen Kinder- und Jugendarbeit und in der Erwachsenenbildung ist nach den Wünschen der Synoden der Umgang mit den Massenmedien einzuïben. Unter den Fachkommissionen, die jede Diözesane Arbeitsgemeinschaft für katholische Erwachsenenbildung einrichten soll, findet sich eine für Medienerziehung. Elternschulung, die Schulung von Medienreferenten und die Weiterbildung von Priestern werden besonders her- 
vorgehoben. Theologen und Religionslehrern sollen medienkundliche Ausbildungsstipendien angeboten werden, umgekehrt ist interessierten Journalisten eine Weiterbildung in kirchlichen und religiösen Fragen zu ermöglichen. Schließlich ist auch an Wettbewerbe zur "mediengerechten Vermittlung bestimmter theologischer Inhalte“ gedacht.

\section{Anmerkungen:}

\section{Zur Zitation der Synodentexte:}

$S$ Salzburger Diözesansynode

Dr. Hans Widrich: Erneuerung der Erzdiözese Salzburg durch lebendige Christengemeinden, Bericht und Dokumentation über die Salzburger Diözesansynode 1968, Salzburg 1969. (Zitate nach Seitenzahlen.)

W Wiener Diözesansynode

Leben und Wirken der Kirche von Wien, Handbuch der Synode 1969-1971, Wien o. J. - Der Text "Kirche und soziale Kommunikation" ist in "Communicatio Socialis" 4 (1971), S. 246-255, dokumentiert.

I Innsbrucker Diözesansynode miteinander für alle, Innsbrucker Diözesansynode 1971-1972, hrsg. vom Bischöflichen Ordinariat Innsbrudk, o. J.

K Kärtner Diözesansynode Bischöfliches Verordnungsblatt der Diözese Gurk/Klagenfurt vom 1. Mai 1972.

P St. Pöltner Diözesansynode Diözesanblatt für die Diözese St. Pölten vom 10. Dezember 1972.

O O Osterreichischer Synodaler Vorgang (Osterreich-Synode)

Osterreichischer Synodaler Vorgang, Dokumentation, Wien 1974. - Der Synodentext "Kirche und Massenmedien" ist in "Communicatio Socialis" 7 (1974), S. 335-360, dokumentiert.

\section{***}

1. O IV/2.2.31.

2. O IV/2.2.32.

3. "Filmschau", Organ der Katholischen Filmkommission für Österreich, 23 Jahrgänge 1951 bis 1972 , Wien.

4. Dr. Richard Emele, 25 Jahre Filmkommission - Rüdkblick und Ausblidk, in: „Filmschau" 41/1972, Seite 7.

5. Inter mirifica 21.

6. Dr. Anton Fellner, 1967-1971 Generalsekretär der Wiener Diözesansynode, war vorher Chefredakteur der Tageszeitung "Neues Osterreich", danach der "Wiener Kirchenzeitung “, ist seit 1975 Leiter der Abteilung Kirche im Fernsehen des ORF. Er war zugleich jahrelang Präsident des "Katholischen Zentrums für Massenkommunikation“" und bis 1975 Vorsitzender der "Katholischen Fernsehkommission für Osterreich". Auch Dr. Walter Schaffelhofer, der Sekretär des "Osterreichischen Synodalen Vorgangs", hat ein Nahverhältnis zum Journalismus und spielt bis heute als Generalsekretär des Verbandes Osterreichischer Zeitungsherausgeber eine wichtige Rolle in der österreichischen Medienpolitik.

7. "multimedia“" Zeitschrift für kritische Medienarbeit, hrsg. vom Katholischen Zentrum für Massenkommunikation seit Januar 1973, Wien.

8. Synode Wien, Information, Bericht, Dokumente, hrsg. vom Generalsekretariat der Wiener Diözesansynode 1967-1971, 8/67, Seite 2 ff. 
9. a. a. O. Seite 4, Film III

10. W 727

11. Synode Wien $7 / 70$, Seite 2

12. W 728

13. $\mathrm{W} 731$

14. Vgl. W 731 mit Communio et progressio 121

15. W 732

16. $O \mathrm{IV} / 3.4$.

17. I I/8

18. P Leitsätze $1-8$, A. Verkündigung

19. W 725

20. O IV/2.1.12.

21. Communio et progressio 11

22. O IV/2.1.11.

23. $O \mathrm{IV} / 2.1 .23$.

24. O IV/2.1.22.

25. $O \mathrm{IV} / 2.2 .13$

43. W 129; in der Erläuterung dazu findet sich ein Katalog von Chancen und Beschränkungen der Verkündigung in den Massenmedien.

44. O IV/3.7.

45. $\mathrm{OIV} / 3.6$.

46. O IV/2.2.22.

47. O IV/5.1.

48. Dr. Franz Stauber, Leiter des Katholischen Bildungswerkes der Diözese Linz, ist Präsident des "Katholischen Zen:rums für Massenkommunikation", Vertreter der Kirchen im Kuratorium des ORF und einer der beiden Kirchenvertreter in Hörer- und Sehervertretung des ORF.

49. O IV/4.1.31.; vgl. dazu auch W 744, 745, P B 20.

50. OIV/5.2.2. Die Zahlen galten für 1974 .

51. OIV/4.3.5. und Erläuterung

52. $\mathrm{OIV} / 3.8$.

\section{Anmerkungen zum Anbang (Synodentexte in Auswabl)}

1. W $127,737,766,767$

О I/8.2.2., IV/4.1.23., 4.1.2.42,, 4.1.2.43., 4.2.3., 4.2.12., 4.3.5., 4.4.1., 5.1.

2. Diözesane Zentren:

W 736

I I/56

K 3.125 .

P B16

Informationsstellen, kathpreß

S 120

W 738

I I/57

K 3.125., 3.321 .

ठ I/62, IV/4.1.21., 4.1.22., 4.1.24.,

4.1.4., 4.2.12.

Medienreferenten

W 744

P B20

O I/63., IV/4.1.31.

Einzelvorschläge

W 739, 757
K 2.22., 3.126.

P A14

O IV/4.1.14, 5.2.5.

3. $\mathrm{S} 123$

W 131, 736

P A15

O IV/4.3.2., 4.3.3., 4.3.5. und Erläuterung, 4.4.3., 4.5.4., 4.5.5., 4.5.6.

4. W $129,130,749,750$

P A9

O IV/4.5.3.

5. W $129,745,747,753,757$

P A 10

O I/64., IV/4.1.21., 4.1.22., 4.1.26., 4.2.2., 4.2.4., 4.2.5., 4.5.1., 4.5.2., 4.5.3.

6. $\mathrm{S} 122,124$

W $766,767,768$

K 2.28., 3.124.

P A12, A11, B19

O III/6.8.1.1.3., 6.8.1.2.5., IV/4.1.31.,

4.4.1., 4.4.1.31., 4.4.3., 4.4.4., 4.5.3. 
After Vatican II, there have been several diocesan, and one national Synods during 1968 to 1974 in Austria. All the diocesan synods, except the ones from Eisenstadt and Linz treated Church and Mass Media (cf. CS 4:1974, 335-360). The author compares and interprets the content of these documents. They show a number of contradictions, but are also courageous sometimes, and hesitant and defensive at others. The reason for this lies mostly in a traditional relation of the Austrian Church towards mass media, but also in the given structures of a hierarchial Church, and in a somehow missing theology. The writers of the two most important documents, the one from Vienna, and the national synod paper originally contemplated towards a theology in the sense of a communicative understanding of the Church. - Some practical proposals of the papers, in the meanwhile, have been carried out so e.g., the erection of a Catholic Centre for Mass Communication on the national level in Vienna, as well as in some dioceses.

\section{RÉSUMÉ}

Après le Concile Vatican II eurent lieu (1968-1974), en Autriche, sept synodes à l'échelle diocésaine et un à l'échelle nationale (= „Osterreichischer Synodaler Vorgang": OSV). Tous les synodes diocésains, à l'exception d'Eisenstadt et de Linz, eurent pour thème „l'Eglise et les mass media“. Le OSV conclut également par un important document sur "l'Eglise et les mass media“. (cf. CS 4:1974, 335-360)! L'auteur compare et interprète les contenus de ces documents. Ils offrent une série de contradictions internes, sont d'une part courageux, d'autre part hésitants et défensifs. Les raisons de cela proviennent d'une part d'un rapport traditionnel entre l'Eglise autrichienne et les mass media, d'autre part des structures hierarchiques prétextées par l'Eglise, mais aussi d'une théologie insuffisante. Les auteurs des deux textes les plus importants (Vienne et OSV) tentèrent à l'origine d'atteindre le but d'une théologie que lauteur nomme „compréhension communicative de l'Eglise“. Quelques propositions pratiques ont en revanche réussies et sont entretemps en partie exécutées, tel que le "Katholische Zentrum für Massenkommunikation" à Vienne (pour l'Autriche) et dans quelques diocèses.

\section{R E S UM E N}

Después del Concilio Vaticano II se celebraron en Austria siete Sínodos Diocesanos y, de 1968 a 1974, un Sínodo General austriaco (= „Osterreichischer Synodaler Vorgang : OSV). Los Sínodos Diocesanos, excepto los de Eisenstadt y Linz, se ocuparon del tema "Iglesia y medios de comunicación social". También el OSV aprobó un amplio documento sobre el mismo tema (cfr. CS 4:1974, 335-360). El autor compara e interpreta el contenido de estos documentos, que revelan una serie de contradicciones internas. Por una parte son audaces, por otra vacilantes y precavidos. Los motivos se encuentran en parte en la actitud tradicional de la iglesia austriaca frente a los medios de comunicación, en parte en las estructuras de la iglesia jerárquica y también en una teología insuficiente. Los autores de los dos textos más importantes (Viena y OSV) propugnan en principio como meta una teología, que el autor califica de "comprensión eclesial de los medios de comunicación". Algunas propuestas prácticas fueron acertadas y realizadas en parte, tales como el "Centro católico para medios de comunicación social" en Viena (para Austria) y en otras diócesis. 